\title{
Quantitative recovery of spores from thermophilic spore papers
}

\author{
P. H. EVERALL AND C. A. MORRIS \\ From the Public Health Laboratory and Department of Microbiology, Mytton Oak Road, \\ Shrewsbury, UK
}

SUMMARY A homogeniser (Stomacher 400) which does not damage bacteria has been used to produce an homogenate of spore papers, from which it was possible to make quantitative assessments of viable spores recoverable from such papers before and after exposure to sterilising procedures.

After being discouraged for many years, the use of spore papers has now regained favour in the absence of satisfactory physical tests for ethylene oxide and formaldehyde sterilising processes. Such tests depend on the inclusion of suitable standard spore preparations in appropriate test pieces (Line and Pickerill, 1973; Mitchell and Alder, 1975) and their examination for spores remaining viable after exposure to the sterilising process. Customary practice is to transfer the spore paper aseptically to tryptone-soya broth and to incubate at $56^{\circ} \mathrm{C}$ for 14 days. As a method this has the disadvantage that growth, if it occurs, may result from one surviving spore or from many; there will even be occasions when growth will result from contaminants introduced at the time of sampling or culture. However, all such 'positive' results will carry equal weight in the assessment of the sterilising process. What would be preferable, though hitherto considered impossible, would be the quantitative recovery of spores from papers so that not only could the initial loading of the papers be checked but also the survival rates could be determined. Kelsey (1961) stated that in spite of the employment of a variety of the then available disintegrative procedures no quantitative recovery technique could be devised because the spores remained caught up in the filter paper fibres. We here describe a simple method for the quantitative recovery of spores from spore paper in which homogenisation (accomplished using a recently introduced machine developed specially for microbiological analyses) is followed by a modification of the surface dropwise counting technique of Miles and Misra (1938).

\section{Material and methods}

SPORE PREPARATIONS

Spore discs, prepared by Southern Group Laboratories, Hither Green Hospital, London, were supplied by the Department of Health and Social Security. A document accompanying these discs referred to them as Batch 05922.

\section{HOMOGENISER}

This is a Stomacher $400^{1}$ (Sharpe and Jackson, 1972). The homogenising action is brought about by two reciprocating paddles, which repeatedly pound the sample contained in a plastic bag. Advantages ciaimed for it are that, unlike some other homogenisers, it does not disrupt bacteria to any extent or cause a significant rise of temperature in the sample.

The homogenisation of each spore paper was performed in a sterile 'bottom-welded' plastic bag (size $10 \times 15 \mathrm{~cm}$ ) to which $10 \mathrm{ml}$ of strength sterile Ringer's solution had been added. Two of these bags could be accommodated in the Stomacher at any one time. The duration of the pounding process was finally fixed at seven minutes, at the end of which the spore papers had, as judged by eye, completely disintegrated.

\section{SURFACE COUNTING}

From the homogenate thus obtained decimal dilutions were prepared in Lemco nutrient broth, chosen as a diluent because of its ability to neutralise formaldehyde (MacKinnon, 1974). Five drops 
$(0.02 \mathrm{ml})$ of each dilution were dropped on to tryptone-soya agar plates (Oxoid Code CM 129 with $1.2 \%$ Agar No. 3 added), which had been previously dried with the lids open in the incubator for a minimum of two hours. After being left undisturbed for at least 30 minutes, during which time the drops were completely absorbed, $7 \mathrm{ml}$ of molten tryptonesoya agar at $60^{\circ} \mathrm{C}$ was poured into each plate so as to overlay the original agar surface. When this second layer of agar had set, the plates were inverted and incubated at $50^{\circ} \mathrm{C}$ for 14 days.

The precision of the techniques was investigated by pooling six homogenates. From this pool 35 sets of decimal dilutions were prepared and surface dropwise counts were performed as described above.

\section{Results}

\section{MICROSCOPY}

Films made from the homogenate produced by 'Stomaching' were stained for spores. Microscopical examination of these preparations showed an even distribution of spores (Fig. 1).

\section{SURFACE VIABLE COUNTS}

Bacillus stearothermophilus growing aerobically on the surface of an agar plate produces large, effuse colonies, which quickly become confluent, thus rendering counting impossible if incubation is con- $\frac{0}{7}$ tinued for more than one or, at the most, two으. days.

The agar overlay diminished colony size so that it became possible to count with ease drops yielding up to 30 colonies. (This procedure is similar in $\frac{\bar{\sigma}}{\bar{\omega}}$ principle to the incubation of counts on organisms $\frac{\vec{\sigma}}{\vec{\sigma}}$ such as Escherichia coli at $30^{\circ} \mathrm{C}$ instead of $37^{\circ} \mathrm{C} \stackrel{\mathrm{O}}{\circ}$ when the ability to count drops containing larger के numbers of uncrowded colonies increases the $\overrightarrow{0}$ precision of the count.) In practice, we usually counted the colonies developing after $2,4,7$, and $\vec{\omega}$ 14 days' incubation and employed the maximum count in the calculation of colony-forming units.? Colony counts increased up to seven days, but very little change took place between seven and 14 days. ir However, it seems justifiable to incubate for the $\vec{N}$ longer period since it is known that shorter periods are not reliable for, e.g., formaldehyde-damaged spores (Cripps et al., 1976). Frequent counts were necessary because occasionally a colony pushing through the surface of the agar will begin to grow in its normal aerobic form, thus crowding out and rendering invisible the smaller, deep colonies (Fig. 2).

The 35 replicates from the pooled homogenate gave a mean count of $2.06 \times 10^{5}$ per ml with a standard deviation of $0.42 \times 10^{5}$.

Counts of colony-forming units obtained from 28

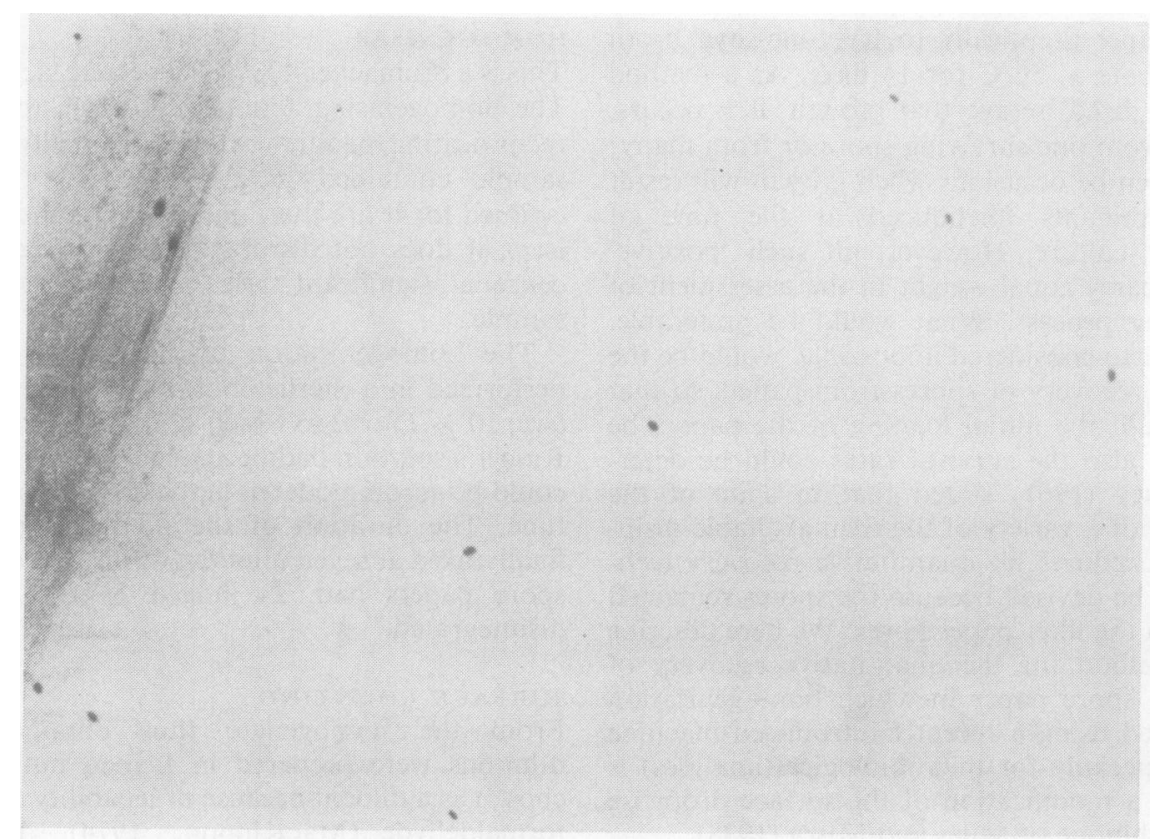

Fig. 1 A film of homogenate after staining for spores with carbol fuchsin. A cellulose fibre is shown on the left with darkly staining spores distributed over the field. 
separate spore discs are shown in the Table.

We have employed this technique to count the spores recoverable from spore papers from various sources currently in use for testing formaldehyde sterilisers and have reported elsewhere the large differences we found (Everall and Morris, 1977). The method described in this note will allow the loading of spore papers to be monitored, but further experiments are necessary to determine to what extent the existing standards for these products, originally developed for autoclaves, are applicable to formaldehyde and similar sterilisers.

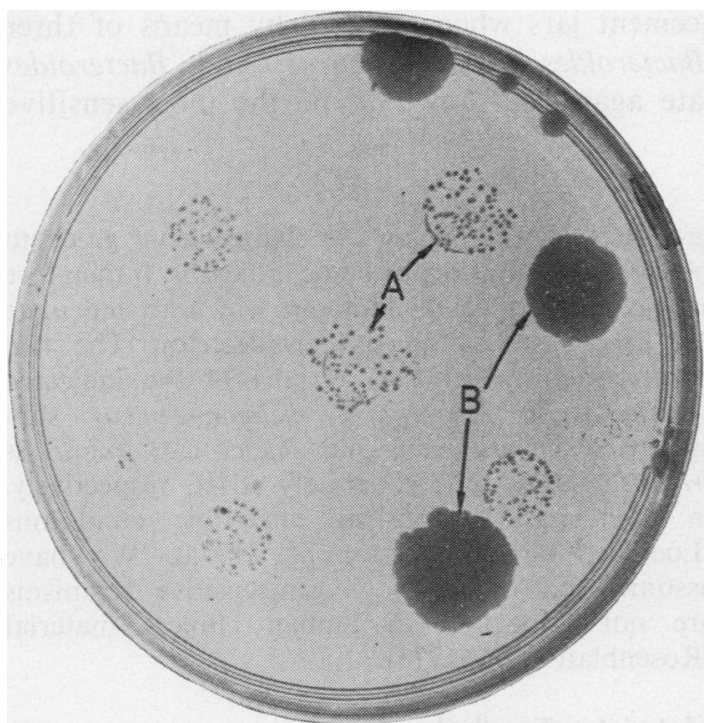

Fig. 2 A seven-day culture on tryptone/soya agar showing both small, deep colonies of $\mathrm{B}$. stearothermophilus $(A)$ and large surface colonies $(B)$ of the same organism.
Table Counts of colony-forming units at $56^{\circ} \mathrm{C}$ for 28 spore discs (all in millions)

\begin{tabular}{llll}
\hline 0.6 & 0.97 & 1.5 & 1.9 \\
0.7 & 1.05 & 1.5 & 1.9 \\
0.73 & 1.1 & 1.5 & 2.0 \\
0.8 & 1.1 & 1.6 & 2.3 \\
0.81 & 1.2 & 1.7 & 2.5 \\
0.9 & 1.2 & 1.7 & 2.5 \\
0.9 & 1.4 & 1.8 & 2.8 \\
Mean count $1.45 \times 10^{6}$ & & &
\end{tabular}

\section{References}

Cripps, N., Deverill, C. E. A., and Ayliffe, G. A. J. (1976). Problems with low-temperature steam and formaldehyde sterilisers. Hospital Engineering, 30, No. 19, 9-11. International Federation Issue.

Everall, P. H., and Morris, C. A. (1977). Testinr of low temperature steam/formaldehyde stariissers with Bacillus stearothermophilus spores. Hospi:al Engineering, 31, No. 6, 3.

Kelsey, J. C. (1961). The testing of sterilizers. 2. Thermophilic spore papers. Journal of Clinical Pathology, 14, 313-319.

Line, S. J., and Pickerill, J. K. (1973). Testing a steamformaldehyde sterilizer for gas penetration efficiency. Journal of Clinical Pathology, 26, 716-720.

MacKinnon, I. H. (1974). The use of inactivators in the evaluation of disinfectants. Journal of Hygiene, 73, 189-195.

Miles, A. A., and Misra, S. S. (1938). The estimation of the bactericidal power of the blood. Journal of Hygiene, 38, 732-749.

Mitchell, J. P., and Alder, V. G. (1975). The disinfection of urological endoscopes. British Journal of Urology, 47, 571-576.

Sharpe, A. N., and Jackson, A. K. (1972). Stomaching: a new concept in bacteriological sample preparation. Applied Microbiology, 24, 175-178.

Requests for reprints to: P. H. Everall, Technical Officer, Public Health Laboratory, Mytton Oak Road, Shrewsbury SY $38 X H$, UK. 